\title{
Frequency of Various Hemoglobinopathies
}

\author{
Sateesh Kumar Lund, Barkha Rani Hindy Mandan Rajput, \\ Dileep Kumar,Mohsin Shafi Rajput, Dr.Ayesha Memon, Dr. Faizan Qaisar \\ Department of Medicine Liaquat University of medical \& Health sciences \\ Department of medicine Glulam Muhammad Maher medical college \\ Department of Medicine Liaquat University of medical \& Health sciences \\ Department of medicine Liaquat University of medical \& Health sciences \\ Department of pathology Liaquat University of medical \& Health sciences \\ Department of Medicine Liaquat University of medical \& Health sciences
}

\begin{abstract}
Objective: To see the pattern of hemoglobinopathies in the blood samples, received for Hb electrophoresis.

Introduction: Hemoglobinopathy is a kind of genetic defect that results in abnormal structure of one of the globin chains of the hemoglobin molecule. Approximately 250 million people (4.5\% of the world population) carry potentially pathological haemoglobinopathy gene. Some of the hemoglobinopathies are due to the production of abnormal hemoglobin proteins. Other types of hemoglobinopathies result from reduced production of hemoglobin proteins that otherwise are normal. We attempted to see the pattern of various hemoglobinopahies in the blood samples of the patients referred to our center for Hb electrophoresis.

Setting: Diagnostic \& Research Lab of Liaquat University of medical \& health sciences, Jamshoro, Pakistan. Methodology: In this retrospective study, we included all the consecutive cases from July 2012 to June 2013, referred to Diagnostic \& Research Lab, for $\mathrm{Hb}$ electrophoresis. Hemoglobin electrophoresis was performed by HPLC Variant II Bio-Rad.

Results: A total of 10,000 samples were received during the study period. $15 \%(1,500 / 10,000)$ subjects had confirmed haemoglobinopathies. Of them $43.2 \%$ ( 661/1530) were males \& $56.7 \%($

869/1530) were females. The most predominant was thalassemia trait $8.5 \%$ (850/10,000)followed by thalassemia major $3.25 \%(325 / 10,000), H b D 1.19 \%$ (119/10,000), HbS $1.26 \%(126 / 10,000), H b E 0.8 \%$ (80/10,000).

Conclusion: This study shows that, prevalence of hemoglobinopathies is not uncommon in our population, Therefore, early detection and characterization of haemoglobinopathies is necessary to reduce the burden of affected births in Pakistan.

Keywords: hemoglobiniopathies, LUMHS, Jamshoro, Pakistan
\end{abstract}

\section{Introduction}

Haemoglobinopathies are the most prevalent genetic defect worldwide, with an estimated 269 million carriers [1]. Globally, the populations of certain regions are at higher risk of having a hae-moglobinopathy [1,2]; while approxi-mately 5\% of the world's population carries a gene for sickle-cell anaemia or thalassaemia, the percentage of carriers can reach $25 \%$ in some regions [3]. A majority of the haemoglobinopathies are not clinically apparent but some produce serious life-threatening diseases and constitute a significant health care burden. These are quantitative (thalassaemia syndromes) or qualitative (variant $\mathrm{Hb}$ ) [4-7]. Thalassaemia syndromes are sub-classified based on the gene involved, i.e. $\alpha$ and $\beta$. These $\alpha-$ and $\beta-$ thalassaemias are further sub-divided into $\alpha+, \beta+$ or $\alpha$, $\beta$ o depending on whether some $(+)$ or no(o) globin protein is produced as a result of the causative mutation.

In Pakistan, where the prevalence of thalassaemia is about 5\%-8\% [8,9], tha-lassaemia continues to be a health care challenge and burden on affected fami-lies and the health care delivery system. The disease runs in families where inter-marriages among relatives are common. It is very important to have reliable detection and identification methods for $\mathrm{Hb}$ variants and $\beta$-thalassaemia trait (heterozygous) because this can lead to the prevention of more severe disorders such as thalassaemia major (homozy-gous) in infants [10]. In recent times successful implementation of national thalassemia screening programmes in neighbouring countries such as the Islamic Republic of Iran and Turkey have shown a steady decrease in newly registered thalassemia cases $[7,11]$.

The objective of the present study was to determine the pattern of haemoglobinopathies diagnosed at the Digital and diagnostic research lab of Liaquat University of medical and health sciences jamshoro/Hyderabad Pakistan July 2012 to June 2013. This is one of the largest private sector diagnostic centers in Hydeabad si, Pakistan. 


\section{Sample}

\section{Methods}

In this retrospective study, we included all the consecutive cases from July 2012 to June 2013, referred to Diagnostic \& Research $\mathrm{Lab}$, for $\mathrm{Hb}$ electrophoresis. Hemoglobin electrophoresis was performed by HPLC Variant II Bio-Rad. The research protocol was approved by the medical research review board of Liaqut University of medical \& Health sciences Jamshoro. All the patients involved in this study were briefed about the objectives of the study and informed consent was obtained from each individual.

\section{Data collection and analysis}

Details of patients' age and sex were recorded. The minimum time elapsed since last blood transfusion, if any, was 3-4 months before the blood sample was taken for analysis. For each patient a $3 \mathrm{~mL}$ intravenous blood sample was collected in EDTA-containing vacutainer blood collection tubes. The samples were subjected to testing within 2 hours of sampling using a fully automated blood cell counter (Sysmax KX-21). Hemoglobin electrophoresis was performed by HPLC Variant II Bio-Rad. The band densities were measured through a Turbo Scan digital densitometric analysis system (Fisher Biotech). An HbA2 value > 3.5\% was considered as a cut-off point for beta-thalassemia trait. The red blood cell indices were compatible with thalassemia trait in all cases where the $\mathrm{HbA} 2$ gene was raised.

The gene frequency of different haemoglobinopathies was estimated using the Hardy-Weinberg equilibrium $\mathrm{p} 2+2 \mathrm{pq}+\mathrm{q} 2=1$, where $\mathrm{p}$ is the frequency of the $\mathrm{A}$ allele in the population and $\mathrm{q}$ is the frequency of the a allele in the population. The frequencies of genotypes in the population are given by: $\mathrm{p} 2$ for genotype AA, 2 pq for genotype Aa, q2 for genotype aa.

\section{Results}

A total of 10,000 samples were received during the study period. $15 \%(1,500 / 10,000)$ subjects had confirmed haemoglobinopathies. Of them $43.2 \%$ ( 661/1530) were males \& $56.7 \%($ $869 / 1530)$ were females. The most predominant was thalassemia trait $8.5 \%(850 / 10,000)$ followed by thalassemia major $3.25 \%$ ( 325/10,000), HbD 1.19\% (119/10,000), HbS $1.26 \%(126 / 10,000)$, HbE 0.8\% $(80 / 10,000)$.The frequencies of different haemoglobinopathies and their respective gene frequencies are shown in chart 1 .

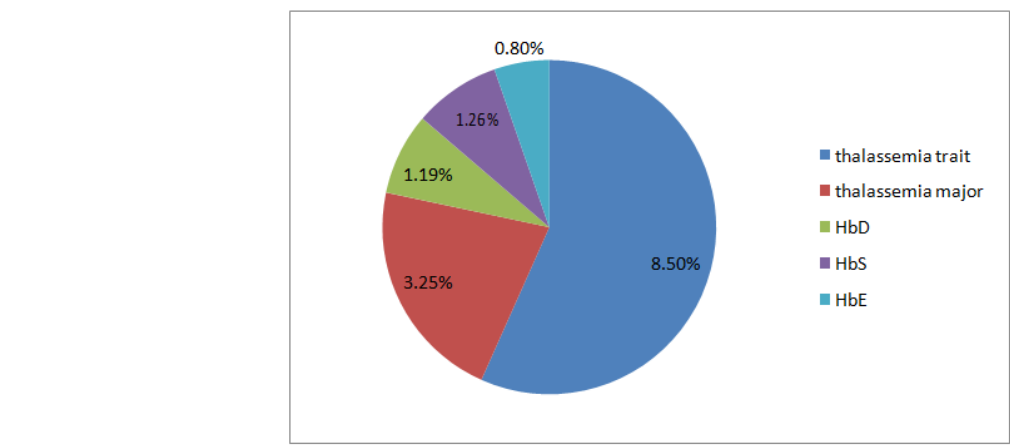

Frequency of various Hemoglobinopathies

\section{Discussion}

The overall frequency of haemoglobinopathies in this study was $15 \%$, which is a little less to a previously presented series of 2000 cases from a referral laboratory in the region revealing that $28.2 \%$ cases presented with haemoglobinopathies [12]. The results ofthis study also support the finding that thalassemia is the most frequent form of haemoglobinopathy in Pakistan [13]. The cumulative percentage of thalassemia genes among individuals having haemoglobinopathies was $11.8 \%$ in this study, compared with $11.85 \%$ in another earlier study [12].This high frequency of thalassemia genes reflects the high regional and geographical prevalence [8,10]. The estimated thalassemia gene frequency in Pakistan is around 5\%-8\%, with 8-10 million carriers and 6000 children born with thalassemia major every year [8,9]. This is partly because of the high ratio of consanguineous cousin marriages and poor access to education and health facilities [14], and also due to lack of a national thalassemia screening and prevention programme $m$ Pakistan. In Pakistan, the concept of thalassemia prevention 1s gammg momentum and a new bill on thalassemia prevention has been put forward in the National Assembly. Our results provide support for these continuing efforts towards early detection and characterization ofhaemoglobinopathies for 
the control and prevention of affected births.

\section{References:}

[1]. Weatherall DJ, Clegg JB. Inherited haemoglobin disorders: an increasing global health problem. Bulletin of the World Health Organization, 2001, 79:704-712.

[2]. Angastiniotis M, Modell B. Global epidemiology of hemo-globin disorders. Annals of the New York Academy of Sciences, 1998, 850:251-269.

[3]. Sickle cell anemia and other hemoglobinopathies. Fact Sheet No. 308. Geneva, World Health Organization, 2006.

[4]. Kutlar F. Diagnostic approach to hemoglobinopathies. Hemo-globin, 2007, 31:243-250.

[5]. Clarke GM, Higgins TN. Laboratory investigation of hemo-globinopathies and thalassemias:review and update. Clinical Chemistry, 2000, 46:1284-1290.

[6]. Colah RB et al. HPLC studies in hemoglobinopathies. Indian Journal of Pediatrics, 2007,74:657-662

[7]. Samavat A, Modell B. Iranian national thalassaemia screen-ing programme. British MedicalJournal, 2004, 329:1134-1137.

[8]. Hafeez $\mathrm{M}$ et al. Regional and ethnic distribution of beta thalas-semia mutations and effect of consanguinity in patients re-ferred for prenatal diagnosis. Journal of the College of Physicians and Surgeons of Pakistan, 2007, 17:144-147.

[9]. Tasleem $\mathrm{S}$ et al. Prenatal diagnosis of beta-thalassaemia by chorionic villous sampling. Journal of the Pakistan Medical Association, 2007, 57:528-531.

[10]. Sachdev R, Dam AR, Tyagi G. Detection of Hb variants and hemoglobinopathies in Indian population using HPLC: report of 2600 cases. Indian Journal of Pathology and Microbiology, 2010, 53:57-62.

[11]. Canatan D et al. Hemoglobinopathy control program in Tur-key. Community Genetics, 2006, 9:124-126.

[12]. Atta $\mathrm{T}$ et al. Frequency of haemoglobin disorders experience at Armed Forces Institute of Pathology. Abstract presented at the 12th Annual Conference of the Pakistan Society of Haematol-ogy, 14-15 February, 2009.

[13]. Khateeb B et al. Genetic diversity of beta-thalassemia muta-tions in Pakistani population. Journal of the Pakistan Medical Association, 2000, 50:293-296.

[14]. Abdullah KN, Azim W, Liaqat J. Beta-thalassemia-institution based analysis of ethnic and geographic distribution, effect of consanguinity and safety of chorionic villus sampling as a diagnostic, tool for pre-natal diagnosis in selected patients. Pakistan Armed Forces Medical Journal, 2010, 4:77-80. 